DOI $10.31489 / 2021$ No2/12-17

UDC 535.34, 535.372, 54.05

\title{
SPECTRAL AND LUMINESCENT PROPERTIES OF CARBON QUANTUM DOTS FUNCTIONALIZED WITH N- AND S-CONTAINING GROUPS
}

\author{
Ibrayev N.Kh., Dzhanabekova R.Kh., Amanzholova G.S.
}

Institute of molecular nanophotonics, E.A Buketov Karaganda University, Karaganda, Kazakhstan, rumiya_@@mail.ru

\begin{abstract}
In the presented work, carbon quantum dots were obtained by microwave synthesis based on citric acid and Lcysteine. The resulting particles were characterized by electron and probe microscopy, dynamic light scattering and Fourier transform infrared spectroscopy. The spectral and luminescent properties were investigated for the initial solution of carbon quantum dots, as well as solutions obtained as a result of dialysis of the synthesized product. It is shown that all samples exhibit the same optical properties. At the same time, the measurement of quantum yields showed that carbon dots that have passed through the dialysis membrane have the best fluorescent ability.
\end{abstract}

Keywords: carbon quantum dots, microwave synthesis, fluorescence, electron microscopy, atomic force microscopy, fluorescence quantum yield.

\section{Introduction}

Carbon quantum dots (CQDs) are nanoparticles less than $10 \mathrm{~nm}$ in size with a carbon structure [1,2]. This new member of the family of carbon nanomaterials is attracting more and more attention due to its promising properties, such as fluorescent radiation, chemical inertness, resistance to photobleaching, high water solubility, low toxicity, biocompatibility, and relatively simple and cheap synthesis methods. All these properties make carbon dots a promising material in such areas as photocatalysis, sensing, bioimaging, solar energy conversion, and the creation of light-emitting diodes [1-4]. There are two main approaches to obtaining CQDs: "top-down" and "bottom-up". The first group includes mechanical grinding, laser ablation, chemical oxidation, electrochemical oxidation. Methods such as microwave synthesis, hydrothermal synthesis, and pyrolysis belong to the second approach to CQDs production. A variety of hydrocarbons, including vegetable and food ones, can be used as raw materials $[2,3]$.

Despite the fact that there are quite a few synthesis methods and applications for CQDs, the question of the source of fluorescence is still unclear. It is believed that the fluorescence of carbon quantum dots is determined by their size and the presence of functional groups [5-7], at the same time, some studies have revealed the contribution of molecular fluorophores to the fluorescence of carbon dots] [8-10]. For example, Essner's group showed that fluorescent impurities are formed as by-products of the synthesis of carbon dots, and CQDs must be sufficiently purified to obtain reliable results [10]. The aim of work is to study the spectral and luminescent properties of carbon dots functionalized with $\mathrm{N}$ - and S-containing groups. To study the effect of the carbon dots size on their optical properties, the synthesized product was purified by dialysis. Comparative studies of the initial solution (without dialysis), the dialysate obtained on the first day, and the solution remaining in the dialysis bag (retentate) were carried out.

\section{Experimental part}

Carbon quantum dots were obtained by microwave synthesis from citric acid (Sigma Aldrich) and Lcysteine (Sigma Aldrich). An equimolar aqueous solution of the starting materials $(5 \mathrm{ml})$ in a borosilicate glass tube was placed in a Monowave 200 microwave reactor (Anton Paar) and heated at $200{ }^{\circ} \mathrm{C}$ for an hour with vigorous stirring. Then the solution was cooled to $70^{\circ} \mathrm{C}$ in a microwave reactor, and then to room temperature in vivo. As a result of the synthesis, a dark brown solution was obtained, which, according to the literature data $[3,5,7]$, indicates the formation of carbon quantum dots. To remove large particles, the resulting solution was centrifuged at 10,000 rpm for 30 minutes. The decantate for further purification was dialyzed using a dialysis bag (MWCO $3.5 \mathrm{kDa}$ ) for 48 hours with periodic replacement of the dialysate with pure water. The structure and sizes of carbon dots were studied using a scanning electron microscope MIRA 3 (TESCAN) using a transmitted electron detector (STEM), an atomic force microscope JSPM-5400 (JEOL), and dynamic light scattering on a submicron particle size analyzer Zetasiser Nano ZS (Malvern). 
FTIR spectra were obtained on an FSM 1201 FT-IR spectrometer (Infraspec). Absorption spectra were measured on a Carry 300 spectrophotometer (Agilent). Luminescence spectra and luminescence excitation spectra were measured on an Eclips spectrofluorimeter (Agilent). The kinetics of fast fluorescence of CQDs solutions was measured on a pulsed spectrofluorometer with picosecond resolution and recording in the timecorrelated photon counting mode (Becker \& Hickl). The fluorescence of the samples was excited using a pulsed semiconductor laser with a generation wavelength $\lambda_{\text {gen }}=375 \mathrm{~nm}$. The kinetic curves were analyzed using the SPCM-Image software (Becker \& Hickl). The fluorescence quantum yield was measured by the absolute method using an AvaSphere 30-REFL integrating sphere and an AvaSpec-ULS2048 spectrometer (Avantes) $[11,12]$.

\section{Results and discussion}

Fig. 1 and Fig. 2 show STEM and AFM images of carbon dots from the initial solution and retentate. In the initial solution particles with sizes from $10 \mathrm{~nm}$ to $400 \mathrm{~nm}$ are observed, the average size is $50 \mathrm{~nm} \pm 10 \mathrm{~nm}$. Large particles are irregular, small and medium particles are rounded. The AFM image shows that the maximum particle height is no more than $1.57 \mathrm{~nm}$. STEM images of CQDs that have not passed through the dialysis membrane show large rounded particles ranging in size from several tens of nanometers to several micrometers and irregularly shaped particles from 10 to $70 \mathrm{~nm}$, from which larger particles are possibly formed. From the images obtained, it can be assumed that small particles are "flakes" resembling graphene structures that can aggregate to form "disk-shaped" particles.

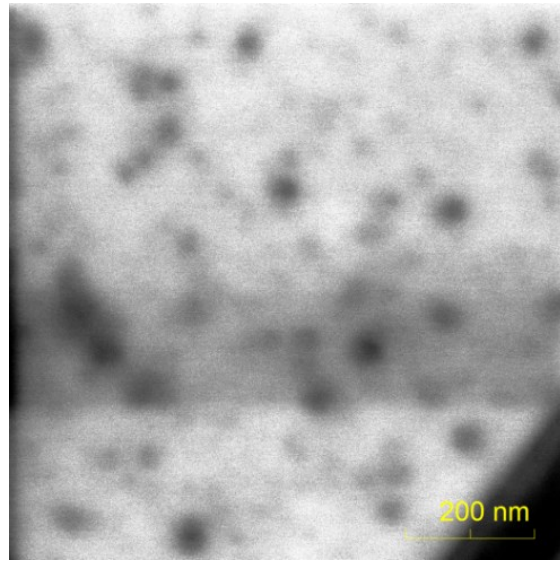

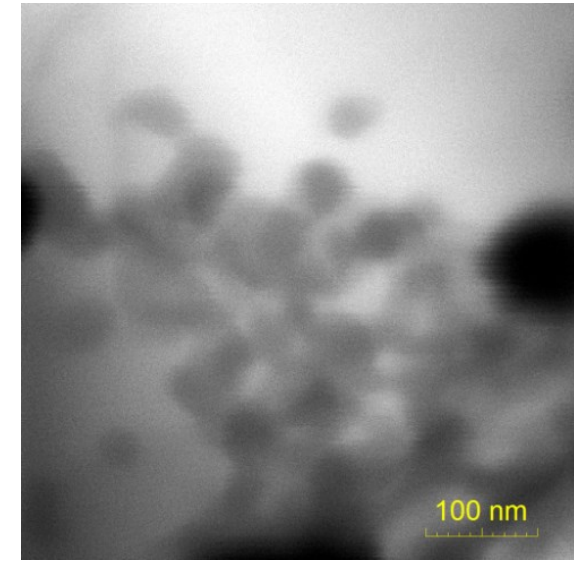

$\mathrm{b}$

Fig.1. STEM images of CQDs from initial solution (a) and retentate (b)

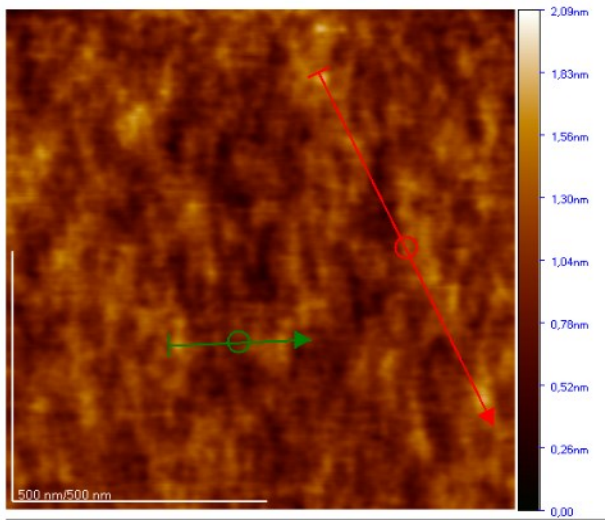

a)

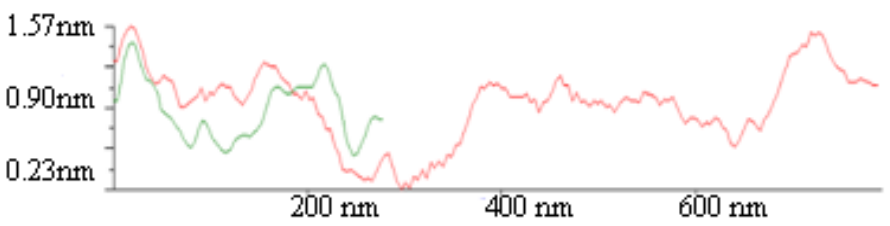

b)

Fig.2. AFM image (a) and surface profile (b) of CQDs from the initial solution

The average size of carbon dots passing through the dialysis membrane was obtained by dynamic light scattering and has a value of $\sim 3.5 \mathrm{~nm}$ (Fig.3). It was not possible to obtain images of particles from the 
dialysate on an SEM using a detector of transmitted electrons due to the insufficient resolution of the microscope.

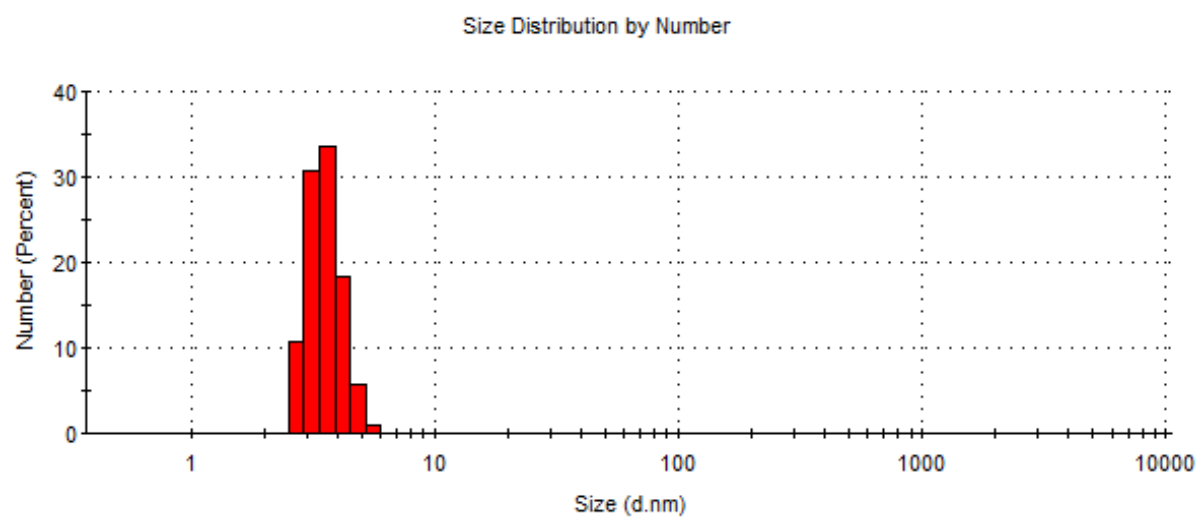

Fig.3. Particle size distribution in dialysate.

Fig. 4 shows the FTIR spectrum of the initial product. The FTIR spectra of the synthesized CQDs agree with the FTIR spectra obtained for the CQDs in [7, 13,14]. The spectra show peaks at 3431 and $3179 \mathrm{~cm}^{-1}$, which refer to the absorption peaks of stretching vibrations of O-H and $\mathrm{NH}$ groups, a weak band at $2928 \mathrm{~cm}^{-1}$ can be caused by stretching vibrations of the C-H bond, the band at $1580 \mathrm{~cm}^{-1}$ is connected with vibrations of the carbon skeleton of the aromatic ring, weak characteristic peaks in the region of $2500-2600 \mathrm{~cm}^{-1}$ correspond $^{-1}$ to the peaks of stretching vibrations of the SH group, the band at $1705 \mathrm{~cm}^{-1}$ is caused by stretching vibrations of the $\mathrm{C}=\mathrm{O}$ bond, the peak at $1400 \mathrm{~cm}^{-1}$ is associated with stretching vibrations of bonds $\mathrm{CN}, \mathrm{NH}$ and $-\mathrm{COO}[7$, $13,14]$.

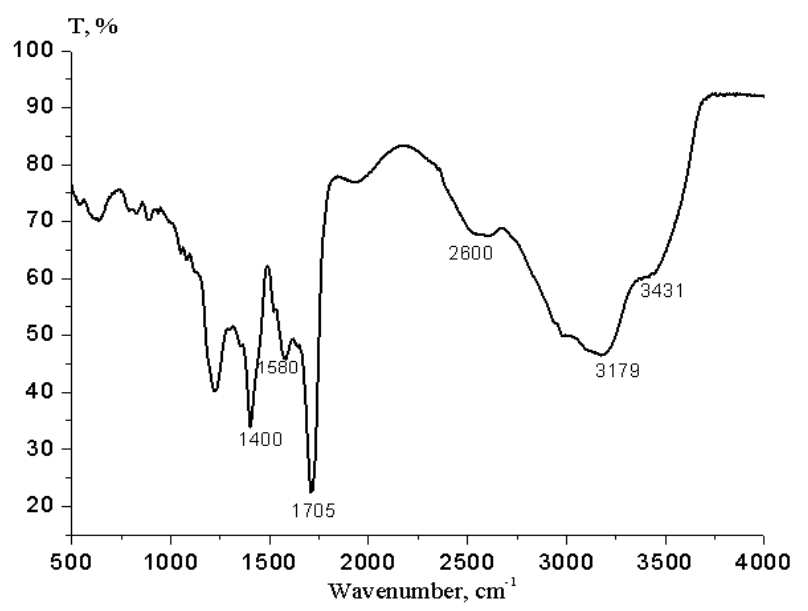

Fig.4. FTIR spectrum of synthesized CQDs

Fig.5. shows the absorption, luminescence, and excitation spectra of the solutions under study. The absorption spectra of all solutions have a band with a maximum at $340-348 \mathrm{~nm}$ and a shoulder at $230-240 \mathrm{~nm}$ and are similar to the absorption spectra of the CQDs obtained in [5, 7]. The shoulder at $230 \mathrm{~nm}$ is attributed to the $\pi-\pi^{*}$ transition $[5,15]$, the absorption band at $340-350 \mathrm{~nm}$ is attributed to the $\mathrm{n}-\pi^{*}$ transition caused by carboxyl, amino and other groups $[5,7,15]$.

It should be noted that the long-wavelength wing of the absorption spectra of the initial solution and retentate extends to the near-IR region of the spectrum. This is especially noticeable for the retentate spectrum. The fluorescence spectra obtained upon excitation at the wavelength of the absorption maximum $(\lambda=350 \mathrm{~nm})$ are located in the wavelength range of $350-650 \mathrm{~nm}$ with a maximum at $\lambda=430 \mathrm{~nm}$. Fluorescence excitation spectra for all solutions correlate with absorption spectra. Table 1 shows the main characteristics of the spectra, from which it follows that the centers of absorption and luminescence in solutions have the same nature. 

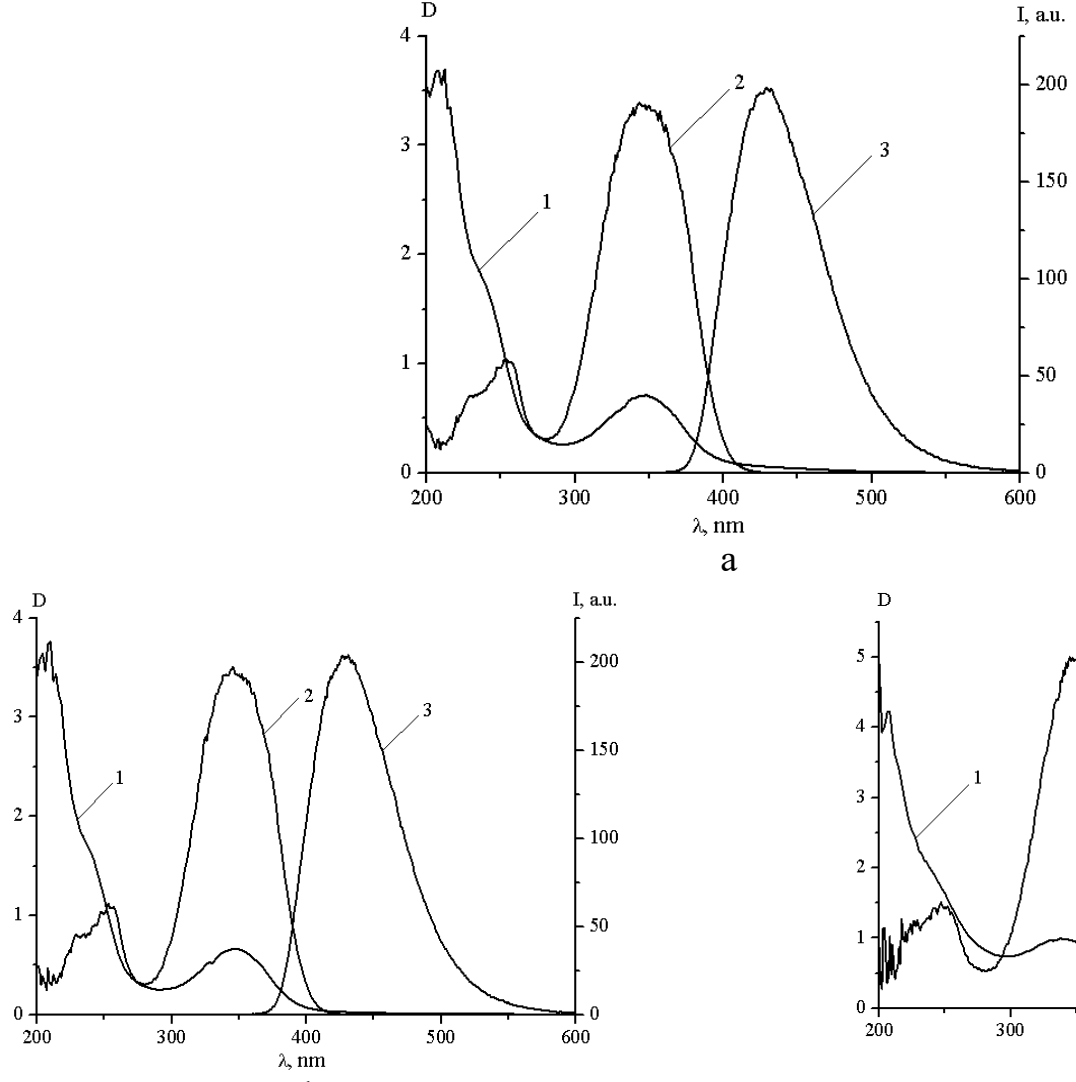

b

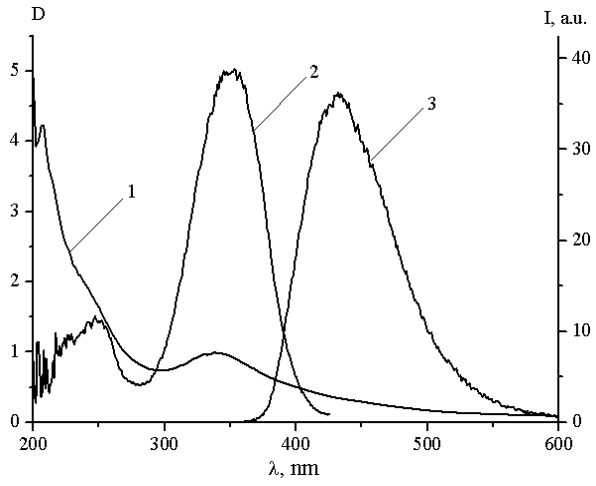

$\mathrm{c}$

Fig.5. Spectra of absorption (1), excitation of fluorescence (2) and fluorescence (3) of the initial solution (a), dialysate (b) and retentate (c).

Table 1. Characteristics of absorption, fluorescence and excitation spectra of aqueous solutions of CQDs

\begin{tabular}{|l|c|c|c|c|c|c|}
\hline Sample & $\begin{array}{c}\lambda_{\downarrow} \max ^{\uparrow} a b s \\
\mathrm{~nm}\end{array}$ & $\begin{array}{c}\text { FWHM (abs), } \\
\mathrm{nm}\end{array}$ & $\begin{array}{c}\lambda_{\downarrow} \max ^{\uparrow} f l, \\
\mathrm{~nm}\end{array}$ & $\begin{array}{c}\text { FWHM (fl), } \\
\mathrm{nm}\end{array}$ & $\begin{array}{c}\lambda_{\downarrow} \max ^{\uparrow} e x, \\
\mathrm{~nm}\end{array}$ & $\begin{array}{c}\text { FWHM (ex), } \\
\mathrm{nm}\end{array}$ \\
\hline Initial solution & 348 & 64 & 430 & 74 & 348 & 69 \\
\hline Dialysate & 348 & 64 & 429 & 74 & 348 & 66 \\
\hline Retentate & 340 & - & 432 & 79 & 350 & 63 \\
\hline
\end{tabular}

The quantum yields obtained by the absolute method are $0.47,0.68$, and 0.03 for the initial solution, dialysate and retentate, respectively. The fluorescence lifetimes measured by the time-correlated photon counting method are $7.8 \mathrm{~ns}$ for the initial solution, $7.3 \mathrm{~ns}$ for dialysate, and $5.9 \mathrm{~ns}$ for retentate. A characteristic feature of the CQDs is the dependence of the luminescence spectra on the excitation wavelength. We measured the fluorescence spectra of the studied solutions under excitation in the range from 350 to $550 \mathrm{~nm}$ (Fig. 6).

The maximum luminescence intensity is observed upon excitation at a wavelength of $350 \mathrm{~nm}$. With increasing excitation wavelength, the fluorescence band shifts to longer wavelengths. In this case, along with a decrease in the luminescence intensity, broadening of the spectra is observed. The shift of the luminescence spectra indicates different luminescence centers, as indicated in $[16,17]$. Luminescence spectra with maxima around 430-450 $\mathrm{nm}$ are the result of deactivation of electronic excitation inside the carbon core of quantum dots and electron-hole recombination. Whereas the red-shifted spectra appear as a result of the capture of electronic excitation by surface traps of different energies or functional groups. This is clearly seen in the absorption and excitation spectra. As can be seen from Fig. 5, it is the carbon core that most intensively absorbs the incident light in the UV region, the band at about $350 \mathrm{~nm}$ is less manifested. In the fluorescence excitation spectrum, on the contrary, the band responsible for functional groups is more manifested. Thus, in synthesized CQDs, it is the surface groups that make the greatest contribution to the total luminescence. 


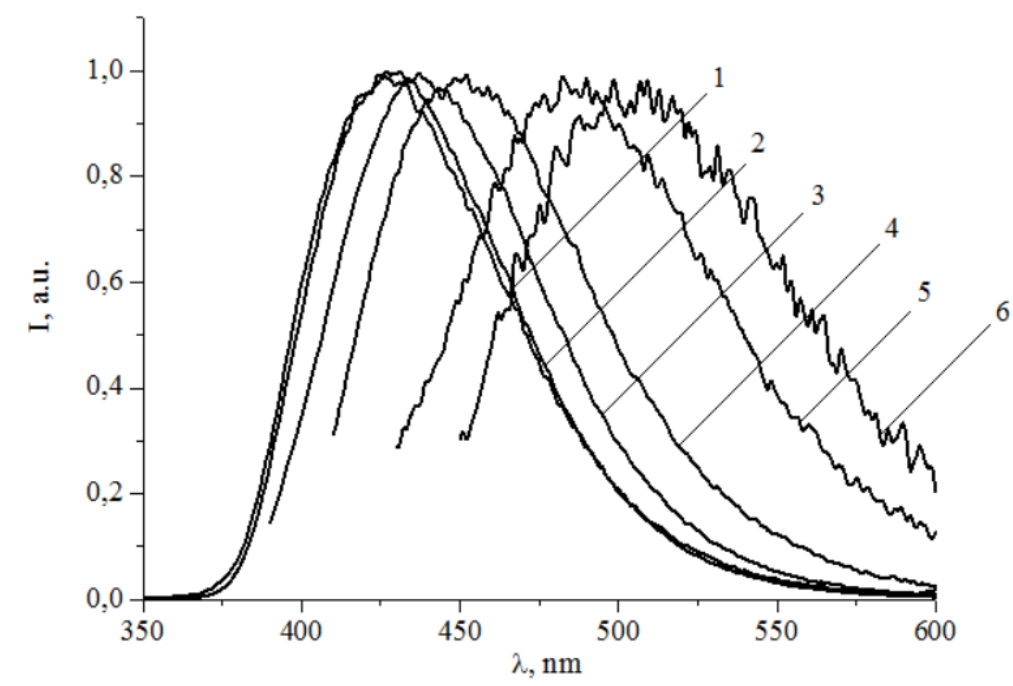

Fig. 6. Normalized CQD fluorescence spectra at different excitation wavelengths: $1-300 \mathrm{~nm} ; 2-350 \mathrm{~nm} ; 3-380 \mathrm{~nm} ; 4-400 \mathrm{~nm} ; 5-420 \mathrm{~nm} ; 6-440 \mathrm{~nm}$

Their composition, as can be seen from the FTIR spectrum, is very diverse, which is responsible for the shift and different intensities of the red-shifted fluorescence of the carbon dots under study.

\section{Conclusion}

Investigations of the structural and spectroscopic properties of carbon quantum dots synthesized by the microwave method have been carried out. It was found by microscopic methods and dynamic light scattering that the resulting particles have the shape of disks, the size of which varies from 3.5 to $400 \mathrm{~nm}$.

FTIR spectra contain characteristic peaks confirming the presence of $\mathrm{O}, \mathrm{N}$ and $\mathrm{S}$-containing functional groups. The positions of the electronic absorption and fluorescence bands are practically the same for the initial solution, dialysate, and retentate. The best luminescent ability with a fluorescence quantum yield of 0.68 is possessed by dialysate carbon dots with a size of $\sim 3.5 \mathrm{~nm}$. With an increase in the excitation wavelength, a long-wavelength shift of the fluorescence spectra occurs, accompanied by a broadening of the spectra. The data obtained can be used in optical nanotechnology to create light-emitting systems based on carbon materials.

The novelty and fundamental difference of the obtained data from similar works is the use of microwave synthesis in the preparation of CQDs from the above-mentioned components. The resulting carbon structures have a rather high quantum yield. This will allow them to be used in various applications, such as laser technology, optoelectronic devices, organic and nanocomposite LEDs and photodetectors, organic photovoltaics. It is also possible to use them as biomarkers and sensors in bioimaging and biophysics.

\section{Acknowledgments}

The authors are grateful to Professor S.E. Kumekov for a fruitful discussion of the results obtained. This research is funded by the Science Committee of the Ministry of Education and Science of the Republic of Kazakhstan (Grant No. AP09259913).

\section{REFERENCES}

1 Molaei M.J. The optical properties and solar energy conversion applications of carbon quantum dots: A review. Solar Energy, 2020, Vol.196, pp. 549-566.

2 Wang Y., Hu A. Carbon quantum dots: synthesis, properties and applications. J. Mater. Chem. C, 2014, Vol.2, pp. 6921-6939.

3 Jhonsi M.A. Carbon Quantum Dots for Bioimaging. Available at: www.intechopen.com/books/state-of-the-art-innano-bioimaging/carbon-quantum-dots-for-bioimaging (June 20, 2018)

4 Yuan T., Yuan F., Li X., et al. Fluorescence-phosphorescence dual emissive carbon nitride quantum dots show 25\% white emission efficiency enabling single-component WLEDs. Chem. Sci., 2019, Vol.10, pp. 9801-9806.

5 Dong Y., Pang H., Yang H.B., et al. Carbon-Based Dots Co-doped with Nitrogen and Sulfur for High Quantum Yield and Excitation-Independent Emission. Angew. Chem. Int. Ed., 2013, Vol.52, pp. 7800-7804. 
6 Qu S., Liu X., Guo X., et al. Amplified Spontaneous Green Emission and Lasing Emission From Carbon Nanoparticles. Adv. Funct. Mater., 2014, Vol.24, pp. 2689-2695.

7 Lin H., Huang J., Ding L. Preparation of carbon dots with high-fluorescence quantum yield and their application in dopamine fluorescence probe and cellular imaging. Journal of Nanomaterials. 2019, Article ID 5037243, pp. 1 - 9. Available at: https://doi.org/10.1155/2019/5037243 (Oct 17, 2019)

8 Song Y., Zhu Sh., Zhang Sh. et al Investigation from chemical structure to photoluminescence mechanism: a type of carbon dots from the pyrolysis of citric acid and an amine. J. Mater. Chem. C, 2015, Vol. 3, pp. 5976-5984.

9 Wang T., Wang A., Wang R., et al Carbon dots with molecular fluorescence and their aplication as a "turn-of" fuorescent probe for ferricyanide detection. Scientific Reports, 2019, Vol.9. Available at: www.nature.com/articles /s41598019-47168-7 (July 24, 2019)

10 Essner J.B., Kist J.A., Polo-Parada L., et al Artifacts and Errors Associated with the Ubiquitous Presence of Fluorescent Impurities in Carbon Nanodots. Chem. Mater. 2018, Vol.30, pp. 1878-1887.

11 Ibrayev N.Kh., Ishchenko A.A., Afanasyev D.A., et al Active laser medium for near-infrared spectral range based on electron-unsymmetrical polymethine dye and silver nanoparticles. Appl. Phys. B. 2019, Vol.125, pp. 1-7.

12 Seliverstova E., Ibrayev N., Omarova G., et al Competitive influence of the plasmon effect and energy transfer between chromophores and $\mathrm{Ag}$ nanoparticles on the fluorescent properties of indopolycarbocyanine dyes. Journal of Luminescence, 2021, Vol. 235, p.118000. DOI:10.1016/j.jlumin.2021.118000

13 Wanga W., Zeng Zh., Zeng G., et al. Sulfur doped carbon quantum dots loaded hollow tubular g-C3N4as novel photocatalyst for destruction of Escherichia coliand tetracycline degradation under visible light. Chemical Engineering Journal, 2019, Vol. 378, p.122132. DOI:10.1016/j.cej.2019.122132

14 Xia Ch., Hai X., Chen X.W., et al. Simultaneously fabrication of free and solidified N, S-doped graphene quantum dots via a facile solvent-free synthesis route forfluorescent detection. Talanta, 2017, Vol.168, pp. 269-278.

15 Wang Y., Kalytchuk S., Zhang Y., et al. Thickness-Dependent Full-Color Emission Tunability in a Flexible Carbon Dot Ionogel. Phys. Chem. Lett., 2014, Vol.5, pp. 1412-1420.

16 Roy P., Po-Cheng Chen P., Periasamy A.P., et al. Photoluminescent carbon nanodots: synthesis, physic-chemical properties and analytical applications. Materials Today, 2015, Vol. 18, No 8, pp. 447-458. DOI:10.1016/j.mattod.2015.04.005

17 Park M., Kim H.S., Yoon H., et al. Controllable Singlet-Triplet Energy Splitting of Graphene Quantum Dots through Oxidation: From Phosphorescence to TADF. Advanced materials, 2020, Vol. 32, No. 31, pp. 2000936 (1-10). DOI: 10.1002/adma.202000936. 